\title{
Accessibility and Visualization for Pulse Meter
}

\author{
Anıl Bölükbaş \\ Master Candidate \\ The Master of Science in Computer Program \\ Jiangsu University
}

\begin{abstract}
Presently a day's heart related illnesses are expanding step by step and there is a requirement for a precise and reasonable pulse checking framework for analyze. The proposed Heart Rate Measuring (HRM) framework is conservative, easy to understand and utilizes optical innovation to distinguish the progression of blood. The framework is intended to detect and gauge the beat by utilizing wearable gadget and afterward showing the outcome on a portable application. This paper clarifies how a solitary chip microcontroller can be utilized to examined heart beat rate flags progressively and gives the data of bradycardia and tachycardia of pulse. This framework can be utilized in the emergency clinics/facilities/research. The target of this work was to develop a model for a pulse oximeter. Given the need, in most medical interventions, to monitories oxygen saturation in peripheral blood $\left(\mathrm{SpO}_{2}\right)$ a pulse oximeter is highly necessary in any working room or intensive care unit. The electronic gadget was built repeating mostly to Integrated Circuit (IC) which communicated with the Personal Computer $(P C)$ what's more including the sensor itself. A module to gauge the carbon dioxide $\left(\mathrm{CO}_{2}\right)$ in a similar principle as pulse oximetry.
\end{abstract}

Keywords: Pulse oximeter, Biomedicine, Arduino, Oxygen saturation, Oximetry design, Microcontroller, Digital Signal Processing.

\section{Introduction}

Heart rate just shows the soundness of our heart. It supports measuring the disorder of cardiovascular system. Humans heart pounds to pump oxygen-rich blood to muscles. Heart rate differs according to the request of powers to absorb oxygen and evacuate carbon dioxide variations such things occur during exercise or sleep. Usually the heart rate which is planned for a resting person is around to $70 \mathrm{bpm}$ for adult males and $75 \mathrm{bpm}$ for adult females. The heart rate monitor is basically a device that takes a sample of heartbeats and estimates the heart beats per minute and the info can simply track the existing heart condition.

Today, patient use various cardiac monitors to measure heart beat rate or pulse. They are differing in term of operational properties, applications and information processing. The heart rate is very important for patient because heart circulate oxygen and blood to whole body. The heart beat rate may differ according to body position. The normal heat beat rate is $60-100 \mathrm{bpm}$, above then $100 \mathrm{bpm}$ is considering fast heart rate and $60 \mathrm{bpm}$ is considering slow heart rate. If abnormalities found the heart rate the person may have disease.

Many top-rated companies offering heart beat rate monitor and presented on the market these devices can also connect with mobile devices as well but the main disadvantage is low accuracy. That's why leading companies like an apple are working on wearable device with high accuracy rate. These devices can declare heart attack predictions which can cause immediate cause blood clots.

Thus, the goal of this development is to make smart watch is low cost and light weight device which can measure highly accurate heartbeat. This device will help and detect heart beat and reduce the death due to the unfortunate medical care. It's practical application based on planned software-hardware solutions for low cost wearable equipment.

- What should we know about our heart rate?

Regardless of whether we're not a competitor, information about our pulse can assist us with observing our wellness level, and it may even assist us with spotting creating medical issues. Our pulse or heartbeat is the occasions our heart thumps every moment. Ordinary pulse shifts from individual to individual. Knowing ours can be a significant heartwellbeing measure.

As our age, changes in the rate and normality of our heartbeat can change and may con note a heart condition or other condition that should be tended to.

\section{Literature Review}


With heart disease increasing day by day, the need for an accurate and affordable heart rate monitor or heart monitor is critical to ensure quality of health. However, most heart rate monitors and environments are expensive and do not follow ergonomics. Our proposed heart rate monitor (HRM) is economical and easy to use, and uses optical technology to measure blood flow through the index finger. Three phases are used to capture pulses on the fingertip, including pulse detection, signal extraction and pulse amplification. This large database offers great opportunities for improving the quality of heart care. Nevertheless, examine the data from these sensors is a ultimatum. The relevant information of $\sim 120,000$ heartbeats per patient per day must be summarized in a human-readable form. Our goal is to make it easier to analyze these bulky data sets. We have developed an open source tool that can be used to create easy-to-interpret diagrams of heart information over long periods of time.[1]

\section{IOT Context Study}

\subsection{IOT System}

The internet of things or then again IOT, is an arrangement of interrelated registering gadgets, mechanical and advanced machines, items, creatures or individuals that are given extraordinary identifiers (UIDs) and the capacity to move information over a system without expecting human-to-human or human-to-PC connection.

\section{- Importance of IOT System:}

Without question, the Internet of Things (IOT) is changing the manner in which individuals and innovation associate. Fuelled by recent propels in systems administration, interchanges, computation, software and equipment advancements. IOT has ventured out of its infancy and is considered as the following leap forward innovation in transforming the Internet into a completely coordinated future internet. However, understanding a system of physical items got to through the Internet acquires a potential danger the shadow of the numerous benefits.[2]

The danger is "security". Given that Wireless Sensor Networks (WSNs) influence the capability of IOT quite efficiently, this paper faces the test of security consideration on a particular, yet expansive, setting of IP-empowered WSNs. In particular, it proposes a novel risk representation device for such networks, called VisIOT. VisIOT is a human-intuitive visual-based anomaly detection framework that is equipped for checking and promptly detecting a few obliterating types of security assaults, including wormhole assaults, and Sybil assaults. In light of a thorough, radial visualization plan, visualization IOT may uncover foes conducting one or various simultaneous assaults against IPempowered WSNs.

\subsection{What is Pulse Meter?}

Pulse Meters utilizes a little gadget called a Pulse oximeter to gauge how a lot of oxygen is in our blood. The gadget doesn't utilize needles to get to the circulatory system. Rather, it just clips on to a fingertip or could be wrist wearable's.

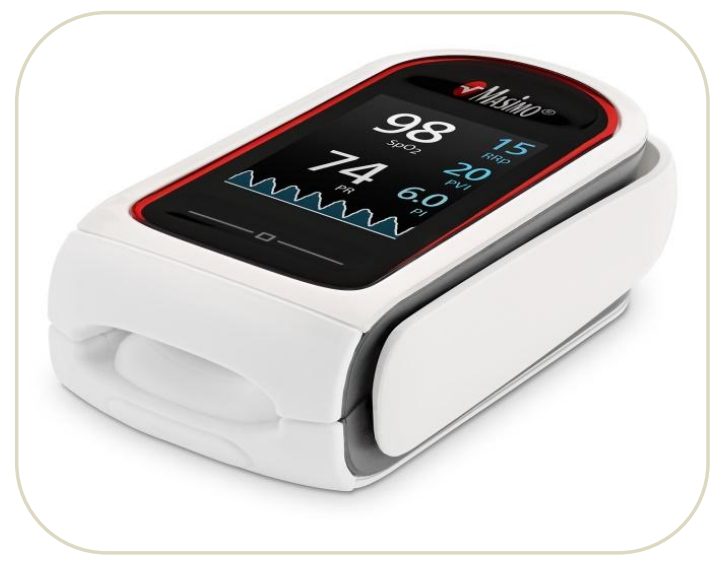

\section{Fig.1}

A light emission goes through the blood in at the tip of your finger and measures the light-retention in the hemoglobin in your blood. It is then ready to ascertain the measure of oxygen in the blood.

What it really gauges is something many refer to as your oxygen immersion level. This thinks about how much oxygen your blood is conveying to the aggregate sum it could be conveying. 
The estimation is communicated as far as a rate. In this way, solid individuals ought to have levels above $89 \%$. Be that as it may, all the more normally they are at 95 to $100 \%$.

Individuals who have COPD can anticipate that their levels should be lower. On the off chance that you are on supplemental oxygen, in any case, the levels should in any case be during the 90s.[3]

\section{Existing approaches:}

There are current methods designed and established for measuring heart rate. At present, the technologies for computing heart rate consist of several approaches using optical and electrical systems. Informally electrical method offers a huge strap around one's chest. But the optical method wants no such strap and it can be used successfully.

Little price heart rate measuring devices were developed using optical technology. In the optical system powerful LED and Light Dependent Resister (LDR) are used to sense pulses. The pulse indications are then amplified through an amplifier circuit and filtered through a band pass filter. The amplified and filtered beat indications are sent to the microcontroller.

Microcontroller checks the analogue indications whether they are effective or not comparing with a normal voltage. Microcontroller sums the heart rate and shows the result in a LCD display. There is another method where infrared Tx and Rx are used. In this system it senses beats, increases the pulses and filters beats by a low pass filter. Lastly, the beat indication is sent to a microcontroller. The microcontroller besides give output linking with a reference voltage. Both the methods give wrong outcome in many situations for the reason that analog signal of beat differs from person to person and the methods fail to adjust analog signal of beats for each person.

- What Is a Normal Pulse?

A typical resting pulse is normally $60-100$ beats for every moment. Our numbers may differ. Kids will in general have higher resting pulses than grown-ups.

\section{- Maximum Heart Rate?}

The maximum heart rate is, on average, the highest our pulse can get. To figure our anticipated greatest pulse, we can use this formula:

1) $220-$ Your Age $=$ forecast Maximum Heart Rate

For example, 40 years an old man forecast maximum heart rate is about 180 beats per minute.

2) Gelish formula: 206.9 - (0.67 $\mathrm{x}$ age $)$

3) Tanaka formula: $208-(0.7 \mathrm{x}$ age $)$

\subsection{Sensors:}

There are different pulse oximeter sensors depending on estimating site (ear, finger probe, temple and multisite) and size of the "patient" (infant, baby, pediatric, or then again adult). There are also disposable sensors (band-wrap or glue) or reusable (band-wrap or clip-on). Examined the impact on test design on precision furthermore, reliability of pulse oximetry in pediatric patients. They compared disposable band-wrap with reusable clip on sensors from three conventional technology pulse oximeters; Nellcor N200, Novametrix 520A and Ohmeda 3700, in 18 children under 12 years of age in a clinical setting in a working room. The saturation values were compared with simultaneous arterial blood gas (hemioximetry). They found that inclination was less than $2 \%$ for any of the test machine combinations and concluded that kind of sensor had little impact on exactness.

Anyway, they pointed out that the children were calmed and in a real pediatric setting a cement sensor may be increasingly practical. Feiner et al. compared clip-on and cement/disposable finger sensors from three new-generation oximeters in 36 adults with different skin pigmentation. The subjects inhaled an air-nitrogen- $\mathrm{CO}_{2}$ blend to accomplish stable low plateau saturation values. All values were compared with blood gas. The mean inclination $\left(\mathrm{SpO}_{2}-\mathrm{SaO}_{2}\right)$ for Masimo Radical (clip-on sensor) for the $70-80 \%$ saturation extend was $2.61 \%$ and $-1.58 \%$ for the disposable. For Nellcor N-595 clip on $2.59 \%$ and $3.6 \%$ for the disposable and for Nonin 9700 clip-on $-0.60 \%$ and for the disposable $2.43 \%$. [ 4 ]

\section{Temperature Sensor:}

The chip on MAX30102 has body heat sensor for scaling the condition according the $\mathrm{SpO}_{2}$ subsystem. The body heat detecting sensor has an implicit resolution of $0.0625^{\circ} \mathrm{C}$. 
The gadget yield information is relatively insensitive to the wavelength of the IR LED, where the Red LED's wavelength is critical to address translation of the information. An $\mathrm{SpO}_{2}$ algorithm utilized with the MAX30102 yield signal can compensate for the related $\mathrm{SpO}_{2}$ blunder with ambient temperature changes.[5]

LED Driver the MAX30102 incorporates Red and IR LED drivers to modulate LED pulses for $\mathrm{SpO}_{2}$ and $\mathrm{HR}$ measurements. The LED current can be modified from 0 to $50 \mathrm{~mA}$ with appropriate supply voltage. The LED pulse width can be modified from $69 \mu$ s to $411 \mu$ s to allow the algorithm to advance $\mathrm{SpO}_{2}$ and $\mathrm{HR}$ precision and force utilization in light of utilization cases.

\subsection{How much a pulse meter is Important?}

Pulse Meters that gauges our oxygen immersion level, or the oxygen levels in our blood. It can quickly identify even little changes in how effectively oxygen is being Pulse Meter is a non invasive and effo conveyed to the limits uttermost from the heart.

The Pulse Meter is a little, cut like gadget that joins to a body part, similar to toes or an ear cartilage. It's most regularly put on a finger, and it's frequently utilized in a basic consideration setting like crisis rooms or clinics. Some experts, for example, pulmonologists, may use it, including Arms legs.

The explanation behind Pulse is to check how well your heart is siphoning oxygen through your body. It may be used to screen the quality of individuals with a condition that can impact blood oxygen levels, especially while they're in the clinical center.

\subsection{Why this system should improve?}

We are living in a new era; people do not have much time to spend in hospitals for their routine checkups. Technology is changing day by day, and now a days everyone wants to calculate each and everything on their fingertips. The invention of smart phones is playing a big role to development of our mankind. By utilizing this technology, we can make changes in our health examine instruments and system to serve the mankind by saving their money and precious time. This exploration is a central standard of the instruments and projects to add to the improvement of wellbeing science. The underlying spotlight on the beat. Heartbeat brought about by the pressure of the heart to send blood around the body, causing pressure on the dividers of the corridors accordingly, vein withdrawal and extension of the cardiovascular cadence. We can feel precisely where the corridor going through or straightforward. Heartbeat is the beat of the heart.

\section{- Purpose of prototype Pulse Meter:}

a) To give heartbeat model to supporting the fundamental clinical science.

b) We study the heartbeat and heartbeat signal that are utilized with equipment and programming.

c) Result of heart can be saved into phone memory.

d) Prototype can be utilized in clinical lab, with 95\% precision, and cheaper price.

e) By Utilizing smart-phone technology get the result on phone screens immediately.

f) By regular checkup and keeping eye on heartbeat situation.

g) By using Arduino technology make the device easy to operate.

h) To make the accessibility for every ordinary people who cannot afford costly environments.

\subsection{What is an ARDUINO?}

Arduino is a gadgets stage dependent on simple to-utilize hardware and works with its operating software. It's proposed for anybody making intuitive ventures.

\section{Arduino Introduction}

This is an open-origin prototyping stage in devices subject to simple to utilize hardware and programming. Quietly, Arduino is a microcontroller based prototyping board that could be used in creating computerized gadgets that can peruse inputs like finger on a catch, address a screen, light on a sensor and so forth and handing it over to yield like turning on a LED, pivoting an engine, playing tunes through a speaker and so forth. The Arduino board can be customized to do anything by essentially programming the microcontroller on board utilizing a lot of directions for which, the Arduino board comprises of a USB attachment to speak with your PC and a lot of association attachments that can be wired to outer gadgets like engines, LEDs and so on. 


\section{How To Communicate With Arduino?}

Arduino sheets are commonly founded on microcontrollers from Atmel Corporation like 8, 16 or 32-piece AVR design based microcontrollers. The significant element of the Arduino sheets is the standard connectors. Utilizing these connectors, we can associate the Arduino board to different gadgets like LEDs or extra modules called shields. The Arduino sheets likewise comprises of on-board voltage controller and gem oscillator. They likewise comprise of USB to sequential connector utilizing which the Arduino board can be customized utilizing USB association.

So as to program the Arduino board, we have to utilize IDE given by Arduino. The Arduino IDE depends on proceeding the program language and supports JAVA coding and $\mathrm{C}++$.

Prior to going further, we will talk about what correspondence is by and large. Correspondence is only trade of data between two gadgets. Here, the data is only information which can be in any way similar to content archives, pictures, sound or video records and so forth. Information can be sent or gotten between two frameworks or gadgets and it is as bits for example 0's and 1's. There are numerous kinds of conventions that are utilized in moving information between two gadgets, however every one of these conventions depend on either Parallel Communication or Serial Communication.

\subsection{ARDUINO Pro Mini}

The Arduino Pro Mini is a microcontroller board based on the it has 14 advanced information/yield pins (of which 6 can be utilized as PWM yields), 6 simple sources of information on board containing resonator, a reset catch and openings for mounting pin headers. A six-pin header can be associated with a FTDI link or Spark fun breakout board to give USB force and correspondence to the board.

The Arduino Pro Mini is proposed for semi-lasting establishment in articles or presentations. The board comes without pre-mounted headers, permitting the utilization of different kinds of connectors or direct patching of wires. The pin design is perfect with the Arduino Mini.

\section{- Memory}

The ATmega328 has $32 \mathrm{kB}$ of blaze memory for putting away code (of which $0.5 \mathrm{kB}$ is utilized for the bootloader). It has $2 \mathrm{kB}$ of SRAM and $1 \mathrm{kBs}$ of EEPROM (which can be perused and composed with the EEPROM library).

\section{- Power Supply:}

The Arduino Pro Mini could be controlled with a FTDI connection or breakout board associated with its 6 pin header, or with a managed supply (contingent upon the model) on the VCC pin. There is a voltage controller ready so it can acknowledge voltage up to $12 \mathrm{VDC}$. In case you're providing unregulated capacity to the board, make certain to interface with the "RAW" nail to not VCC.

The power pins are as per the following:

RAW: For providing a crude voltage to the board.

VCC :The directed 3.3 or 5 volt supply.

GND : Ground pins.

\subsection{Input and Output}

Every one of the 14 advanced pins on the Pro Mini can be utilized as an info or yield, utilizing pin mode, digital write, and digital read capacities. They work at 3.3 or 5 volts (contingent upon the model). Each pin can give or get a limit of $40 \mathrm{~mA}$ and has an inner draw up resistor (detached as a matter of course) of 20-50 k Ohms. Moreover, some pins have specific capacity and abilities.

\subsection{Problems:}

Oximeter have limitations which may result in mistaken readings. Because of the sigmoid state of the bull hemoglobin separation bend, oximetry may not recognize hypoxemia in patients with high arterial oxygen tension levels. Conventional pulse oximeters can recognize only two substances: decreased hemoglobin and oxyhemoglobin. It expects that dyshemoglobins for example carboxy-hemoglobin $(\mathrm{COHb})$ and methemoglobin $(\mathrm{MetHb})$ are absent. Studies demonstrated that the presence of elevated levels of $\mathrm{COHb}$ and MetHb could influence the exactness of $\mathrm{SpO}_{2}$ readings.In healthy volunteers, the exactness of a multiwavelength oximeter (Masimo Rainbow Pulse meter; Masimo Corporation, Irvine, CA, USA) in estimating dyshemoglobins was evaluated by inciting carboxyhemoglobin and 
methemoglobinemia (levels extend from $0 \%$ to $12 \%$ ). Predisposition between COHb levels estimated with the Pulse meter and $\mathrm{COHb}$ levels estimated with the laboratory $\mathrm{CO}$-oximeter (standard strategy) was $-1.22 \%$; the comparing exactness was $2.19 \%$.[6]

Inclination \pm accuracy of MetHB estimated with the pulse CO-oximeter and MetHb estimated with the laboratory COoximeter was $0.0 \% \pm 0.45 \%$. The precision of Pulse meter in estimating levels was also evaluated during hypoxia.

In healthy volunteers, the pulse $\mathrm{CO}$-oximeter was exact in estimating $\mathrm{COHb}$ at a $\mathrm{SaO}_{2}$ of less than $95 \%$ (inclination of $-0.7 \%$ and accuracy of $4.0 \%$ ); in any case, when the $\mathrm{SaO}_{2}$ dipped under $85 \%$, the pulse $\mathrm{CO}$-oximeter was unable to gauge $\mathrm{COHb}$ levels. In patients evaluated in the emergency department with suspected carbon monoxide harming, the predisposition between pulse $\mathrm{CO}$-oximetric measurement of $\mathrm{COHb}$ and laboratory $\mathrm{CO}$-oximetric measurement of $\mathrm{COHb}$ was less than $3 \%$.

The limits of agreement between the measurements nonetheless, were large ( $-11.6 \%$ to $14.14 \%)$, leading a few creators to conclude that these new pulse CO-oximeters may not be utilized interchangeably with standard laboratory measurements of $\mathrm{COHb}$. Erroneous readings with pulse oximetry have been accounted for with intravenous colors utilized for analytic purposes, low perfusion expresses (that is, low cardiac yield, vasoconstriction, and hypothermia), pigmented subjects and in patients with weakness.[7]

Because the two wavelengths (660 and $940 \mathrm{~nm}$ ) that pulse oximeters use to gauge $\mathrm{SpO} 2$ can be created by different ambient light sources, the presence of such sources could deliver false $\mathrm{SpO} 2$ readings. The largest difference in SpO2 between the control condition (that is, complete haziness) and any of the five light sources was less than 5\%. Nail polish can meddle with pulse oximetry readings.

\section{Methodology}

The developed device is wearable armlet that is mounted on the patient's arm shown in the figure. The main part of this project is hardware and software. The max30102 is attached with Arduino pro mini ATmega328 the size of Arduino pro mini is very small that's why it is best option to use this board max30102 module is used to detect heart rate pulse of humans or patients. The working principle behind the Sensor is Photo plethysmography. According to the principle, the varieties in the volume of blood in a body part is estimated by the varieties in the power of the light transmit through that body part. This method polishes light on the skin and the perfusion of the blood is measured. One of the practical features of this approach is that it is possible to distinguish between the light reflected by the blood of a vein (produces an AC output) and other components of the body such as skeletons and muscles (produces a DC output).This method polishes light on the skin and the perfusion of the blood is measured. One of the practical features of this approach is that it is possible to distinguish between the light reflected by the blood of a vein (produces an AC output) and other components of the body such as skeletons and muscles (produces a DC output). The photo-diode in the sensor at that point changes over the light to current that we can use as justifiable information. To counter problems for example, skin tone differences LEDs with various frequencies are utilized. In the MAX30102 there is an additional green LED for this reason. The heartbeat sensor module in the design of the prototype was nominated low cost heartbeat sensor for Arduino. [10]

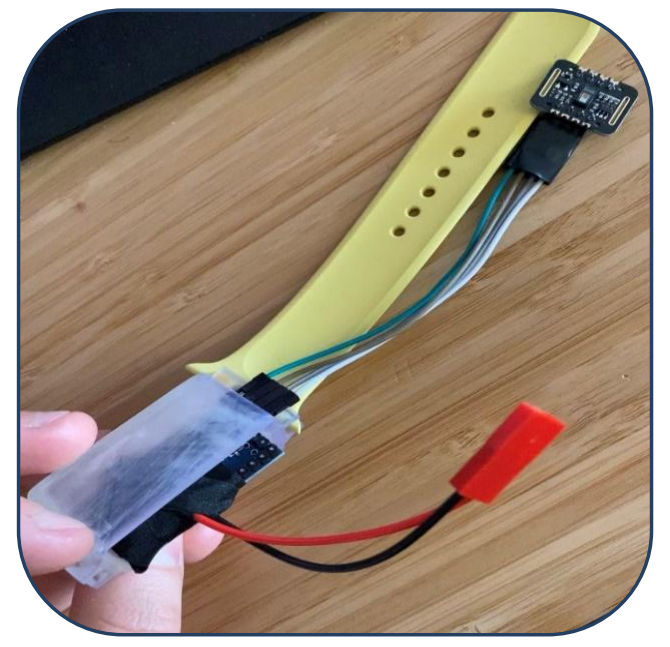

Fig2: prototype wearable device 
This module will convert heartbeat reading is converted to human-readable format using Arduino ide after converting this reading HC-05 module will send this data on mobile application.HC-05 is Bluetooth module used for communication between device and mobile application. For backup there is small size of battery of 500mah is used and TP4056 module for charging battery which is USB type b so user can easily charge this device.

The above figure shows the circuit scheme of prototype wearable device. Where we can see, how our device will work. Also, we can see how ARDUINO PRO MINIis connected to Bluetooth board then batteries. Below figure shows the exact connection scheme and we can understand it easily.

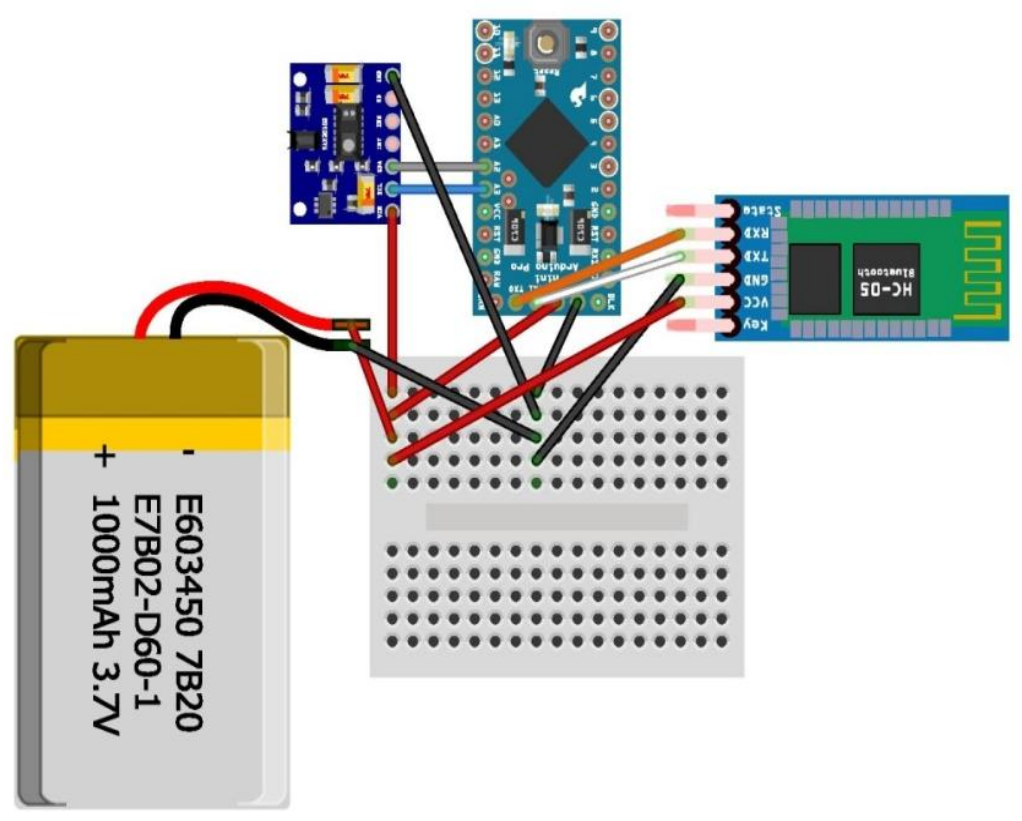

Fig - 3 The circuit diagram of wearable device

\subsection{Data Processing}

A Remote Health Monitoring System includes of three principle parts, for example, information detecting module, information preparing module and information correspondence module. The information detecting module comprises of heartbeat sensor which detects the adjustments in the particular physiological parameters. The data is then passed on to the Arduino genius scaled down microcontroller of information handling module.

The information handling module inspects the information signals. The commotion signals are shifted and if the prepared worth surpasses than ordinary worth. The correspondence module is utilized to move information among individual and hardware. This has fundamental segments, for example, the message, the sender and the collector, the medium and the convention by which the message is sent to the portable application.

Right now, utilized the Arduino pro Mini advancement board which have ATmega328 microcontroller and the microcontroller is utilized to dissect the quantities of heart thumps per minute. The determined qualities originating from the sensor is sent to the android application by means of HC-05 Bluetooth module. A string persistent takes the simple sign of heart condition and sends the determined readings to the android application. In the event that the sign voltage is over the edge voltage, at that point it is estimated as a heartbeat is identified and tallies up to 30 pulsates. A clock whole the ideal opportunity for 30 thumps and figures pulse.

In our undertaking, advancement of android application called "Heart screen", is a vigorous bit. We screen the heartbeat rate through this application. When the heartbeat is estimated the microcontroller then these readings sends the outcome to the versatile application through the HC-05 Bluetooth module. Out of nowhere the present state of the patient is appeared in the application. In this way, anybody can basically comprehend the present circumstance of the patient and can make moment move.[8] 


\subsection{Communication:}

The Arduino Pro Mini has different workplaces for talking with a PC, another Arduino, or distinctive microcontrollers. The ATmega328 gives UART TTL consecutive correspondence, which is available on mechanized pins 0 (RX) and 1 (TX). The Arduino programming joins a consecutive screen which licenses clear scholarly data to be sent to and from the Arduino board by methods for a USB affiliation.

A Software Serial library mulls over consecutive correspondence on any of the Pro Mini's electronic pins. The ATmega328 also supports I2C (TWI) and SPI correspondence. The Arduino programming consolidates a wire library to improve usage of the I2C transport; see the reference for fine distinction to use the SPI correspondence.

\section{How to install Arduino and Programming.}

Arrange or introduce the required USB drivers and furthermore the essential arrangement of the IDE for the Arduino board to be perceived by the IDE. Right now, will proceed with the Arduino IDE by investigating its highlights, understanding the essential activity and transferring our first program to the Arduino UNO board associate Arduino UNO board to the PC by means of the USB link and select the fitting board and COM port in the event that it isn't done as of now. There is no requirement for any outer force supply to the Arduino board as it draws the fundamental force from the USB association. The projects in Arduino condition are called as Sketches. In the Arduino IDE, the void area featured in the picture beneath is called as Text Editor. This space can be utilized for composing all the code for our Arduino sheets. In the event that there is an error in the sketch like a missing semicolon or an unclosed section, the gathering stops by then and the message window calls attention to mistake alongside its sort and area. Utilizing this, we can address the error.

The second symbol neighboring the verify symbol is the upload symbol. When the aggregation of the sketch is effective, at that point we can transfer the sketch utilizing this symbol. There is a console easy route Ctrl + $U$ to transfer the aggregated sketch. At whatever point we click on the upload symbol, the sketch is assembled before transferring.

As our sketch is aggregated effectively with no blunders, we can proceed with transferring the sketch. At the point when we click the transfer symbol, the sketch begins transferring and the micro controller on the Arduino board gets modified.

During this time, the TX and RX LEDs on the Arduino UNO load up squint as a sign that the information is being transmitted through the sequential port. When the sketch is effectively transferred, we can see a message in the message window as Done Uploading.

On the off chance that the drivers are effectively introduced and the framework perceives the gadget, we can discover the Arduino UNO board associated with one of the COM ports in the "Ports" classification in the gadget administrator.

It is imperative to take note of the quantity of the COM port to which Arduino is associated. This is on the grounds that we may associate numerous gadgets and even various Arduino sheets and every gadget can be separated by the COM port.

In the event that the Arduino UNO isn't recorded in Ports yet in "Different gadgets" class, at that point the drivers of the USB to sequential connector are not effectively introduced. Right now, can physically introduce the drivers for this, right snap on the gadget in different gadgets class and select update driver programming choice.

At that point select the subsequent choice for example peruse my PC for introducing drivers situated on the PC. Go to the area where Arduino is introduced and go to driver's organizer in that. In that envelope, select the "arduino-org.inf" record. At that point Windows will introduce the important drivers from here.

Dispatch the Arduino IDE either by choosing the work area alternate route or by heading off to the envelope where it is introduced. At the point when the IDE is propelled, a clear sketch will open. With this progression, we have effectively introduced the Arduino IDE, associated the Arduino UNO to the PC and made the fundamental settings according to the board and port being used.

Since we have some thought regarding sequential correspondence, we compose a program for correspondence among Arduino and the PC.

For ExampleIntbuttonstate $=0$; void setup ()$\{$ pinmode $(13$, OUTPUT $)$;pinmode $(10 \quad$, INPUT $) ;\}$ void $\operatorname{loop}()\{$ buttonstate=digitalRead $(10) ;$ if $($ buttonstate==LOW $)\{\operatorname{digitalWrite}(13, \mathrm{HIGH}) ;\}$ else $\{$ digitalWriteI13,LOW $) ;\}\}$ 


\section{Working}

Java based animation created on processing software. Calculations are made based on RPM between 60-120.

\section{Visualization code}

float count;float $\min \_$speed $=0.5$;float $\max \_$speed $=4$;float min_pulse $=60$; float max_pulse $=120$;float $\mathrm{s}=0.5$; void setup() \{size(1080, 720);fill(0);noStroke(); colorMode(HSB, $\quad 360, \quad$ 100, 100);rectMode(CENTER);frameRate(30);noiseDetail(2, 0.9);noCursor();count = 0;startSerial();textAlign(CENTER, CENTER); \}Java based animation created on Processing software. Calculations are made based on RPM between 60120.

Effects of visualization such as color and speed changes by RPM shown in Figure a, Figure b 2 and Figure c.

Fig b.

Fig a.

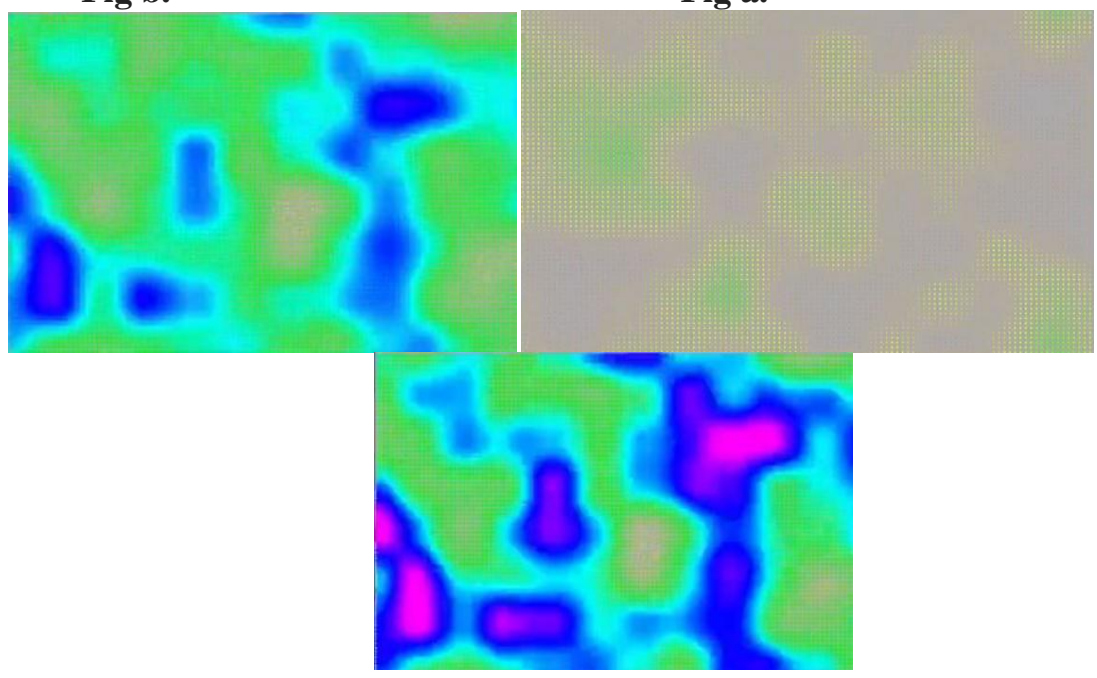

Fig c.

\subsection{Modes of Operation}

The HC-05 Bluetooth Module can be arranged in two methods of activity: Command Mode and Data Mode. In Command Mode, you can speak with the Bluetooth module through AT Commands for arranging different settings and parameters of the Module like get the firmware data, change UART Baud Rate, change module name, set it as either Master or slave and so on. A significant point about HC-05 Module is that it very well may be designed as Master or Slave in a correspondence pair. So as to choose both of the modes, you have to enact the Command Mode and sent fitting at commands. Going to the Data Mode, right now, module is utilized for speaking with other bluetooth gadget for example information move occurs right now.

Name: HC-05

password: 1234 (or 0000)

Type: Slave

Mode: Data

Baud Rate: 9600 with 8 information bits, no equality and 1 stop bit

To show the association between HC-05 Module and Arduino, I have structured a straightforward circuit.

The point of this circuit is to interface the Bluetooth Module with Arduino, Pair the Bluetooth Module with an Android Phone, send information from Android Phone to the Bluetooth Module utilizing a basic app, read the information from Bluetooth Module through Arduino lastly, show the information and control a gadget dependent on the information.

\subsection{HC-05 Bluetooth module}

The HC-05 is an exceptionally cool module which can include two-way (full-duplex) remote usefulness to your tasks. We can utilize this module to impart between two micro controllers like Arduino or speak with any gadget with bluetooth usefulness like a phone or laptop. There are numerous android applications that are as of now accessible which makes this procedure significantly simpler. The module speaks with the assistance of USART at 9600 baud rate henceforth it is anything but difficult to interface with any micro controller that underpins USART. 
We can likewise design the default estimations of the module by utilizing the order mode. So program that we are searching for a Wireless module that could move information from our PC or cell phone to micro controller or the other route around then this module might be the right choice for us.

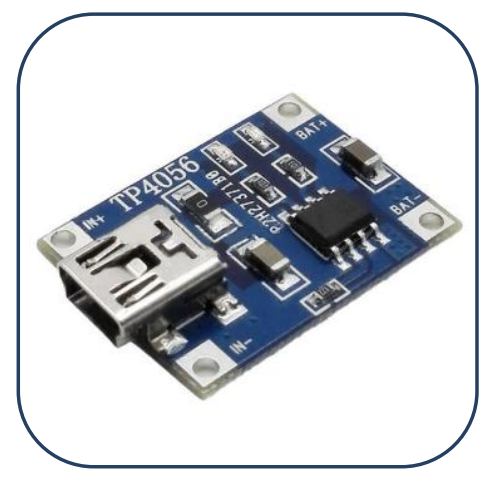

\section{Fig.4}

Sequential Bluetooth module for Arduino and different microcontrollers

Working Voltage: $4 \mathrm{~V}$ to $6 \mathrm{~V}$ (Typically $+5 \mathrm{~V}$ )

Working Current: $30 \mathrm{~mA}$

Range: $<100 \mathrm{~m}$

Works with Serial correspondence (USART) and TTL perfect

Follows IEEE 802.15.1 institutionalized convention

Utilizations Frequency-Hopping Spread range (FHSS)

Can work in Master, Slave or Master/Slave mode

Can be effectively interfaced with Laptop or Mobile telephones with Bluetooth

Upheld baud rate: 9600,19200,38400,57600,115200,230400,460800

\subsection{The TP4056 charging module:}

The TP4056 is a finished consistent current/steady voltage direct charger for single cell lithium-particle batteries. Its SOP bundle and low outside segment check make the TP4056 obviously appropriate for versatile applications. Besides, the TP4056 can work inside USB and divider connector.

This little module is ideal for charging single cell 3.7V 1 Ah or higher LiPo cells, for example, 16550s that don't have their own security circuit. Based around the TP4056 charger IC and DW01 Deep Drain Battery Protection IC this module offers $1 \mathrm{~A}$ charge current. The module cuts off charging current when wrapped up.

Besides when the battery voltage dips under $2.4 \mathrm{~V}$ the assurance IC will turn the heap off to shield the cell from running at excessively low of a voltage - and furthermore secures against over-voltage and invert extremity association anyway please check you have it associated effectively the first run through.

- Under Voltage Cut off/Deep Drain Protection

- Over Current Protection

- Adjustable Current Limit through R-Programming

- Over-voltage charging insurance

- USB Micro Connector (with all the more generally accessible USB links)

- LED Status Indicator for Charging/Full charge status checking

\subsection{Programming}

The ATmega328 on the Arduino Pro Mini come pre burned with a boot loader that licenses us to move new code to it without the use of an outside gear engineer. It confers using the first STK500 show references C, header reports. 


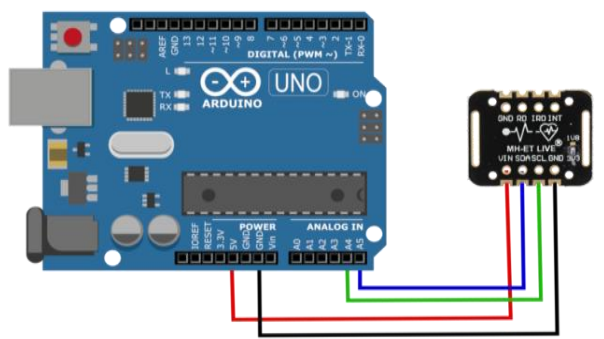

\section{Fig.5}

/* This code is for Arduino Mega only*/ char data =0; voidsetup() \{ Serial.begin(9600); Serial1.begin(9600); pinMode (8, OUTPUT); \} voidloop ()$\quad\left\{\operatorname{if}(\right.$ Seriall $\operatorname{available}()>0) \quad\left\{\right.$ data $=$ Serial1.read () ; if $\left(\right.$ data $=={ }^{\prime} 1$ ') \{ Serial.print(data); Serial.print("=> LED ON"); Serial.print("In"); /*Serial1.print("LED is ON"); Serial1.print("In");*/ digitalWrite(8, HIGH); \} elseif(data == '0') \{ Serial.print(data); Serial.print("=> LED OFF"); Serial.print("In"); /*Serial1.print("LED is OFF"); Serial1.print("In");*/ digitalWrite(8, LOW); \} \} \}

\section{Code}

\section{Programming Analysis}

Presently, we will see the hypothesis behind the catch press. As referenced before, the tenth pin of the Arduino is pulled high and therefore, it consistently identifies rationale HIGH. Additionally, the catch is associated between pin 10 and ground. Consequently, if the catch is squeezed, the association between the pin 10 of Arduino and ground is shut and thus, Arduino will identify a rationale LOW on the tenth pin. With the assistance of this progress from Logic HIGH to Logic LOW, we can distinguish if the catch is squeezed or not and hence turn the LED on or off. As the circuit is effectively depicted with its structure and hypothesis, the following stage is to compose the program or sketch for this circuit and rationale in the Arduino IDE. For composing the code effectively, we have to follow the accompanying advances. Introduce a pin as yield for the LED.

Instate a pin as contribution for the catch or switch. Recognize the status of the catch. Turn the LED on or off. In this way, the initial step is to instate a (Pin 13) of the Arduino as yield for LED and another (Pin 10) as contribution for button. As we have found in the past instructional exercise, we have to utilize the capacity pin Mode to instate a pin as info or yield. Henceforth, compose the pin Mode capacities for both LED and catch pin in the arrangement work.

When we introduced the pins for LED and switch, the subsequent stage is to check for the status of the switch. So as to do this, we have to utilize a capacity called digitalRead ();

digitalRead, as the name indicates, is a capacity which is utilized to peruse esteems from advanced pins for example it peruses for rationale LOW or HIGH at the computerized pins.

The sentence structure of digitalRead work is digitalRead (pin no);

In the language structure, pin no shows the quantity of the computerized pin from which you need to peruse the advanced information. The digitalRead capacity can return two qualities: HIGH or LOW. On the off chance that the digitalRead distinguishes $5 \mathrm{~V}$ at the pin, at that point HIGH is returned. So also, on the off chance that the digitalRead distinguishes $0 \mathrm{~V}$ at the pin, at that point $\mathrm{LOW}$ is returned.

Going to our circuit, we have to peruse the status of the catch pin for example tenth pin. Make a variable before arrangement and name it as "buttonstate", with the goal that the worth returned by the digitalRead capacity can be caught. Presently tuned in work, appoint the arrival estimation of the digitalRead of tenth pin to the buttonstate variable. 


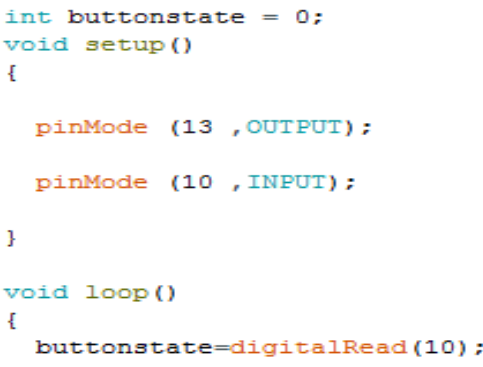

With this progression, we currently have caught the status of the catch in a variable. The following stage is to control the LED. So as to control the LED for example either turn it on or off, we have to utilize a contingent explanation called if and else.

\subsection{Arduino MAX30102:}

Pulse meter screens the oxygen saturation in blood by estimating the size of reflected red and infrared light. Pulse Meters can also rough pulse by analyzing the time arrangement reaction of the reflected red and infrared light. The MAX30102 pulse oximeter is an Arduino-compatible and inexpensive sensor that licenses calculation of pulse utilizing the technique portrayed previously. The MAX30102 sensor will be presented along with several inside and out analyses of the red and infrared reflection information that will be utilized to calculate parameters, for example, pulse and oxygen saturation in blood.

The MAX30102 is an incorporated pulse oximetry and pulse screen module. It includes internal LEDs, photograph indicators, optical elements, and low-commotion electronic with ambient light dismissal. The MAX30102 gives a complete framework solution to facilitate the design-in process or mobile and wearable gadgets. The MAX30102 works on a single $1.8 \mathrm{~V}$ force supply furthermore, a different $3.3 \mathrm{~V}$ force supply for the internal LEDs. Communication is through a standard I2C-compatible interface. The module can be closed down through programming with zero backup current, allowing the force rails to remain controlled consistently.

Applications

- Wearable Devices

- Fitness Assistant Devices

- Smartphone

- Tablets

\section{Detailed Description:}

The MAX30102 is a complete pulse oximetry and pulse sensor framework solution module designed for the requesting requirements of wearable gadgets. The gadget maintains a small solution size without sacrificing optical or electrical execution. Minimal external equipment components are required for combination into a wearable framework. The MAX30102 is fully adjustable through programming registers, what's more, the digital yield information can be put away in a 32-profound FIFO inside the IC. The FIFO allows the MAX30102 to be associated with a microcontroller or processor on a common transport, where the information isn't being perused continuously from the MAX30102's registers.

The $\mathrm{SpO}_{2}$ subsystem of the MAX30102 contains ambient light cancellation (ALC), a consistent time sigma-delta ADC, and a restrictive discrete time filter. The ALC has an internal Track/Hold circuit to cancel ambient light and increment the viable powerful range. The ALC can cancel up to $200 \mu \mathrm{A}$ of ambient current. The internal ADC is a constant time over sampling sigma-delta converter with 18-piece resolution. The ADC sampling rate is $10.24 \mathrm{MHz}$. The ADC yield information rate can be modified from 50sps (samples every second) to $3200 \mathrm{sps}$.

The MAX30102 highlights an I2C/SMBus-compatible, 2-wire serial interface comprising of a serial information line (SDA) and a serial clock line (SCL). SDA and SCL facilitate communication between the MAX30102 and the ace at clock rates up to $400 \mathrm{kHz}$. The ACE generates SCL and starts information move on the transport. The ACE gadget composes information to the MAX30102 by transmitting the correct slave address followed by information. Each transmitter sequence is encircled by a START (S) or REPEATED START (Sr) condition and a STOP (P) condition. 
An ace perusing information from the MAX30102 transmits the correct slave address followed by a arrangement of nine SCL pulses

\section{Discussion Research and Findings}

Working with critically ill children with congenital coronary illness, we had a infant with transposition of the extraordinary veins (TGA) without an arterial line. The pediatric cardiologist on call was to decide if the infant required a Rashkind-septostomy late Friday evening or not. He looked at the saturation screen at the pediatric cardiac ward displaying post ductal oxygen saturation (optimal measurement quality) of $85 \%$. By coincidence this infant was included in the examine venture with channel dependent babies, and had both pre-and post-ductal saturation estimated with another generation oximeter, Masimo SET at the same time (paper I). Post ductal saturation with the newgeneration oximeter was $67 \%$ (optimal measurement quality). Lacking an arterial line for blood gas analysis, the specialist on call settled on the choice to play out a Rashkind-septostomy.

Had he believed the $85 \%$ perusing, a septostomy would not have been performed. We were worried about the tremendous differences in optimal quality readings from the different oximeters and lacking an arterial line we never discovered the "genuine saturation" right now. This perception lead to the possibility of the fourth paper, the clinical observational concentrate in our cardiac unit comparing our equipment with both blood gas (golden standard) and another generation oximeter. We really felt that we needed to see whether this was a "one time" finding or not and that we needed to make a "quality control" on our equipment observing or children with CHD. Today, a couple of years later I'm glad to see that the checking equipment at our pediatric cardiac ward have all been changed to the newgeneration technology. The medical technology improves all the time and working in a hospital, especially with wiped out children I believe one must keep oneself refreshed with the technology. It is an essential to utilize validated research results in a valuable manner. Research and ordinary hospital schedules must be closely associated with the goal that implementation of improvements can happen inside a reasonable time allotment.

\section{Survey Insights}

\section{IOT Concepts for Pulse Meters}

\section{Introduction}

A total of 30 individuals (aged between $18 \& 54$ ) provided their responses to the IOT Concepts for Pulse meters survey, conducted on 10/03/2020.

In this report, we present:

1. An introduction to Likert Scale for Survey response analysis

2. Response analysis per question:

(a) Do you think this visualization is intelligible

(b) Would you buy or interact with this system in future

(c) Would you recommend this system to a friend

(d) How likely do you think that this system is useful

(e) Do you think the system is entertaining

3. The distribution of participants across age categories (Count \& Percentage)

4. Main Findings

5. References

An Introduction to Likert Scale for survey response analysis:

In survey research, Likert scales are the most commonly used type of scale. A Likert scale is a psychological measurement device that is used to gauge attitudes, values, and opinions. It functions by having a person complete a questionnaire that requires them to indicate the extent to which they agree or disagree with a series of statements. The Likert scale is named after its creator, Rensis Likert, who developed it in 1932. [9]

Hence, instead of only allowing 'yes' or 'no' answers, the attitude scale lets respondents add nuance to their answers. The more nuanced responses can be, the more detailed and insight rich the analyses can be. [10]

The Likert scale usually comprises five or seven options (an odd number of choices) as the respondents are offered a central (neutral) category. In certain cases, the Likert scale can use an even number of choices; when no central(neutral) category is offered. These choices can take different labeling such as probability,likelihood, agreement \& satisfaction regarding different statements/survey questions 
In the below figure, we find examples of 5 scale likert choiceIn the below figure, we find examples of 4 scale likert choices ProbabilityNote: In this report, 4 questions are 5-scale and 1 question is 4-scale; as we shall see.

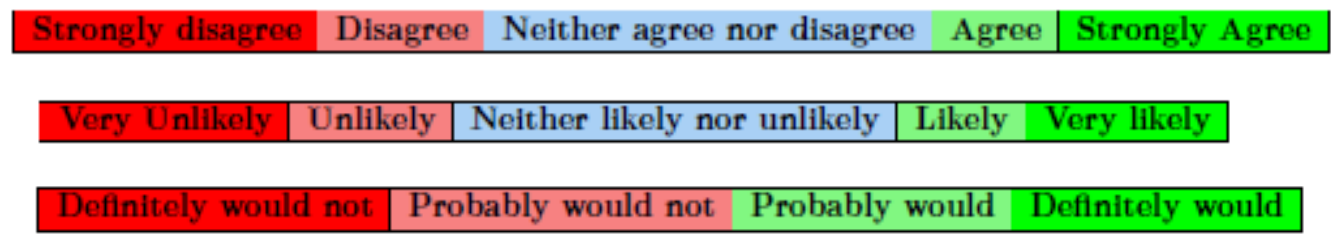

\section{Response analysis per question}

30 Responses (per question). Questions are ordered based on the overall participants' feedback; from the least positive to the most positive (based on the sum of 2 percentages related to the lower 2 choices on thescale for each question) Do you think this visualization is intelligible?

\section{Responses; 5-Scale}

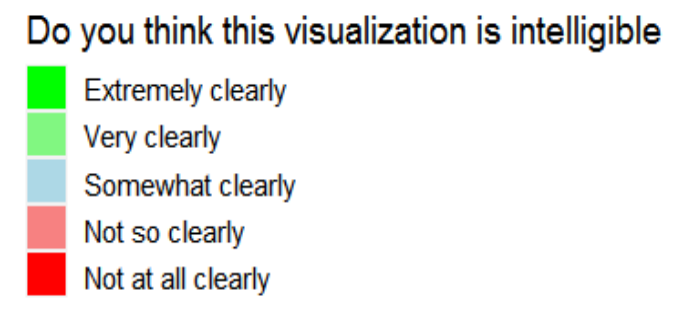

Results Summary:

- $0 \%$ of participants have chosen (Not at all clearly)

- 3\% of participants have chosen (Not so clearly)

- $13 \%$ of participants were uncertain and have chosen (Somewhat clearly)

- $43 \%$ of participants have chosen (Very clearly)

- $40 \%$ of participants have selected (Extremely clearly)

In the below chart, we show an overall representation of the results related to the above question where the graph is centered at the neutral category (if it exists); as follows:

- Percentage on the Left is the sum of the negative (lowest 2) categories on the scale

- Percentage on the Right is the sum of the positive (highest 2) categories on the scale

- Percentage on the Middle is related to the central(neutral) category.

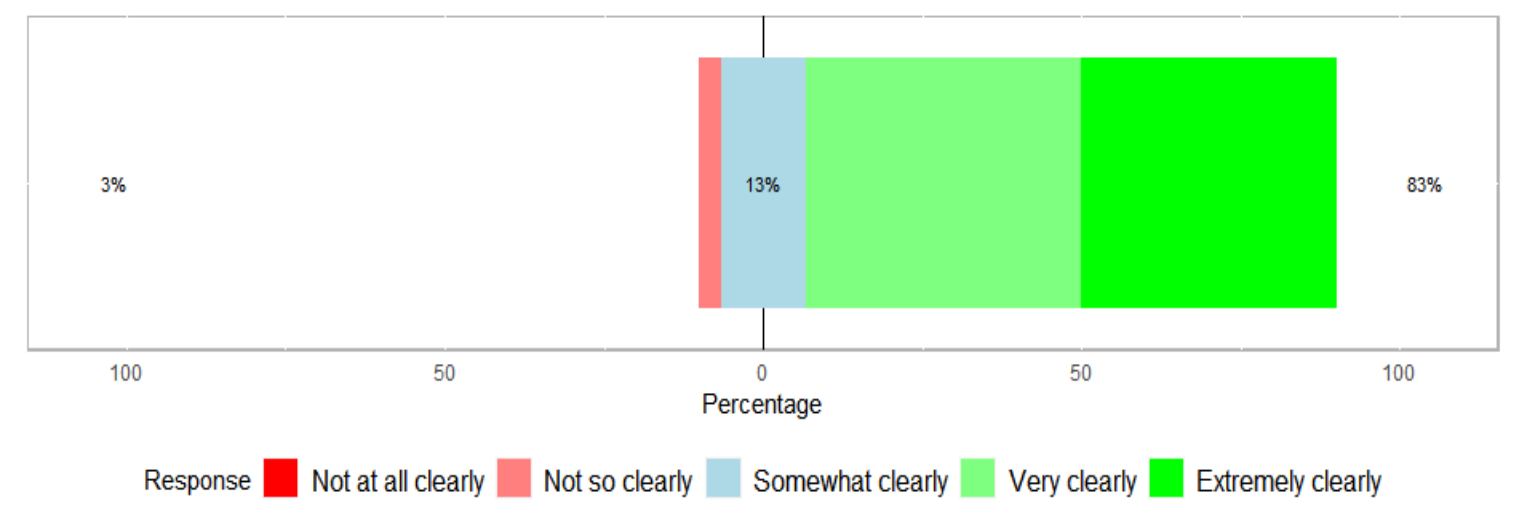


Would you buy or interact with this system in future?

30 Responses; 5-Scale

Would you buy or interact with this system in future
Extremely desirable
Very desirable
Somewhat desirable
Not so desirable
Not at all desirable

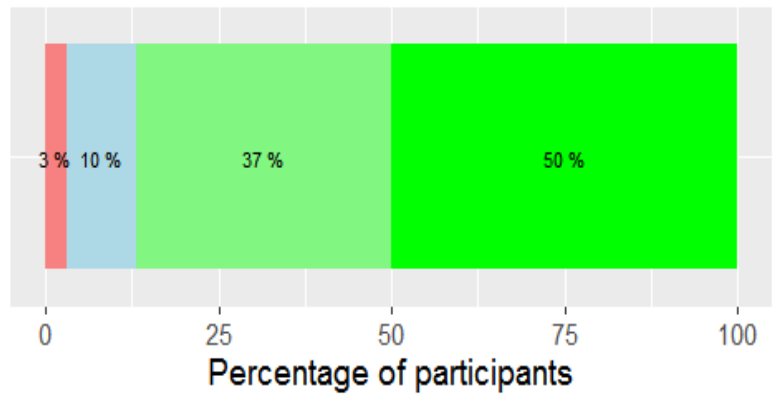

\section{Results Summary:}

- $0 \%$ of participants have chosen (Not at all desirable)

- $3 \%$ of participants have chosen (Not so desirable)

- $10 \%$ of participants were uncertain and have chosen (Somewhat desirable)

- $37 \%$ of participants have chosen (Very desirable)

- 50\% of participants have selected (Extremely desirable)

In the below chart, we show an overall representation of the results related to the above question wherethe graph is centered at the neutral category (if it exists); as follows:

- Percentage on the Left is the sum of the negative (lowest 2) categories on the scale

- Percentage on the Right is the sum of the positive (highest 2) categories on the scale

- Percentage on the Middle is related to the central(neutral) category.

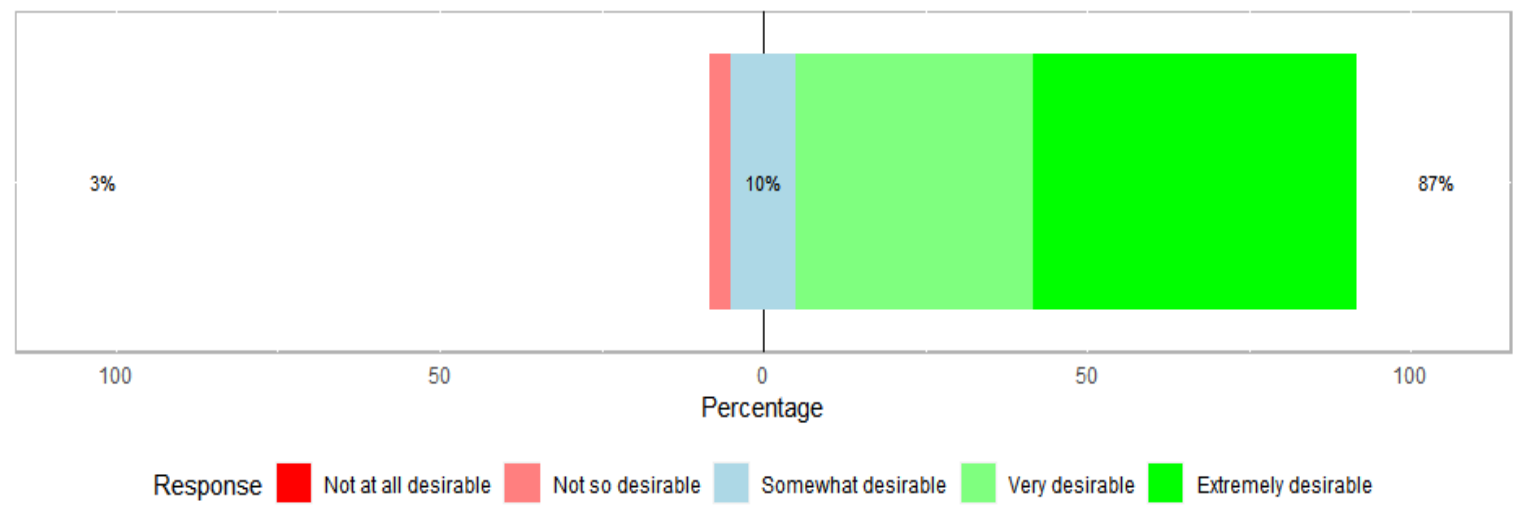

Would you recommend this system to a friend?

\section{Responses; 4-Scale}

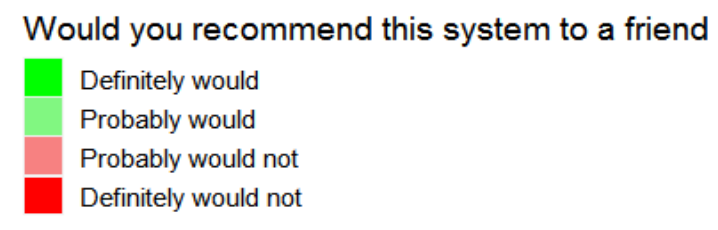

\section{Results Summary:}

- $0 \%$ of participants have chosen (Not at all desirable)

- 3\% of participants have chosen (Not so desirable)

- $0 \%$ of participants were uncertain and have chosen (Somewhat desirable) 
- 30\% of participants have chosen (Very desirable)

- $67 \%$ of participants have selected (Extremely desirable)

In the below chart, we show an overall representation of the results related to the above question where the graph is centered at the neutral category (if it exists); as follows:

- Percentage on the Left is the sum of the negative (lowest 2) categories on the scale

- Percentage on the Right is the sum of the positive (highest 2) categories on the scale

- Percentage on the Middle is related to the central(neutral) category.

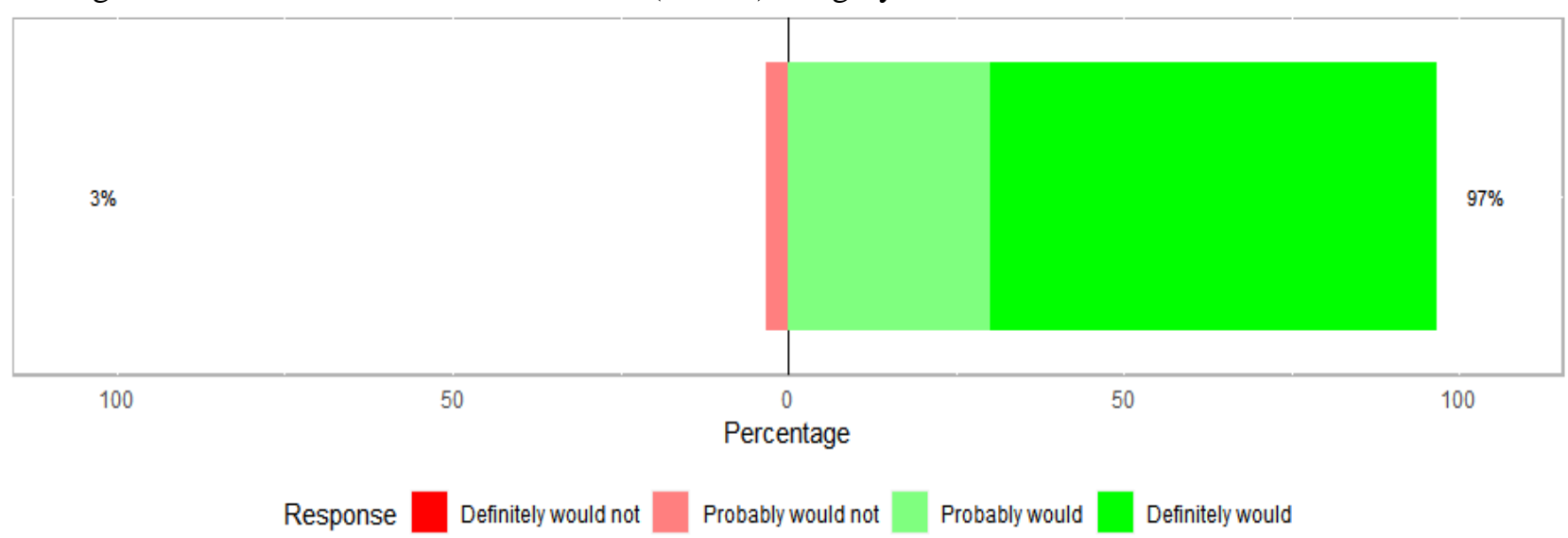

\section{How likely do you think that this system is useful?} 30 Responses; 5-Scale

How likely do you think that this system is useful

Strongly agree

Agree

Neither agree nor disagree

Disagree

Strongly disagree

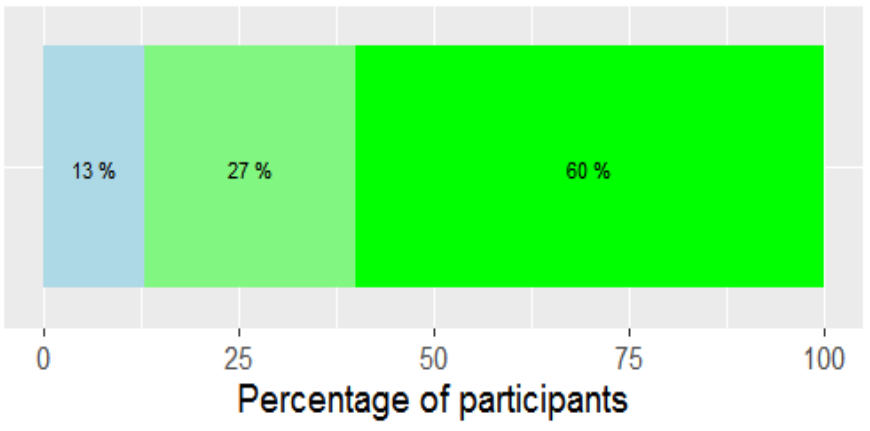

\section{Results Summary:}

- 0\% of participants have chosen (Strongly disagree)

- $0 \%$ of participants have chosen (disagree)

- $13 \%$ of participants have chosen (Neither agree nor disagree)

- $27 \%$ of participants have selected (agree)

- $60 \%$ of participants have selected (Strongly agree)

In the below chart, we show an overall representation of the results related to the above question where the graph is centered at the neutral category (if it exists); as follows:

- Percentage on the Left is the sum of the negative (lowest 2) categories on the scale

- Percentage on the Right is the sum of the positive (highest 2) categories on the scale

- Percentage on the Middle is related to the central(neutral) category 


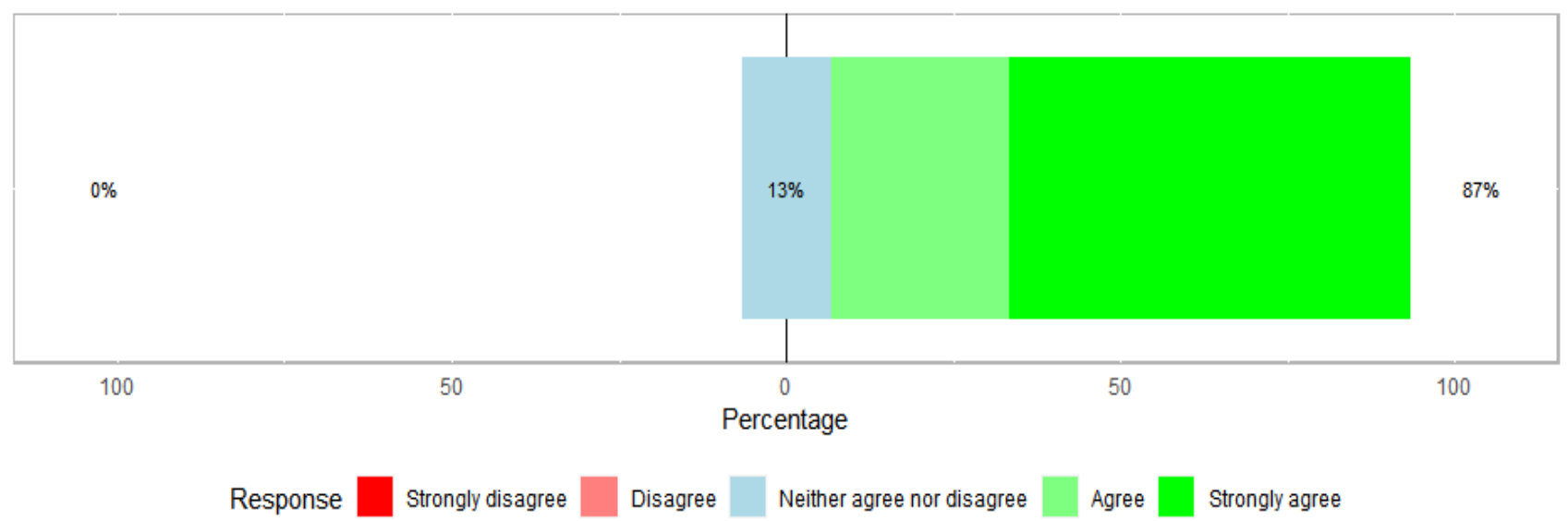

Do you think the system is entertaining?

\section{Responses; 5-Scale}

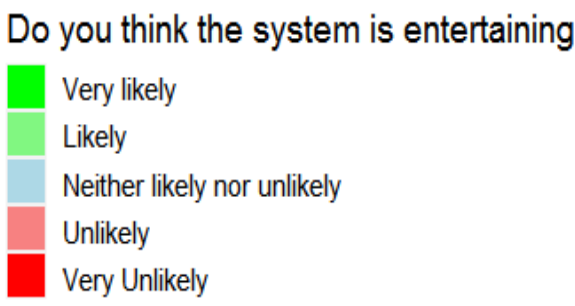

\section{Results Summary:}

- $0 \%$ of participants have chosen (Very Likely)

- $0 \%$ of participants have chosen (Unlikely)

- 3\% of participants have chosen (Neither likely nor unlikely)

- $40 \%$ of participants have selected (Likely)

- 57\% of participants have selected (Very likely)

In the below chart, we show an overall representation of the results related to the above question wherethe graph is centered at the neutral category (if it exists); as follows:

- Percentage on the Left is the sum of the negative (lowest 2) categories on the scale

- Percentage on the Right is the sum of the positive (highest 2) categories on the scale

- Percentage on the Middle is related to the central(neutral) category

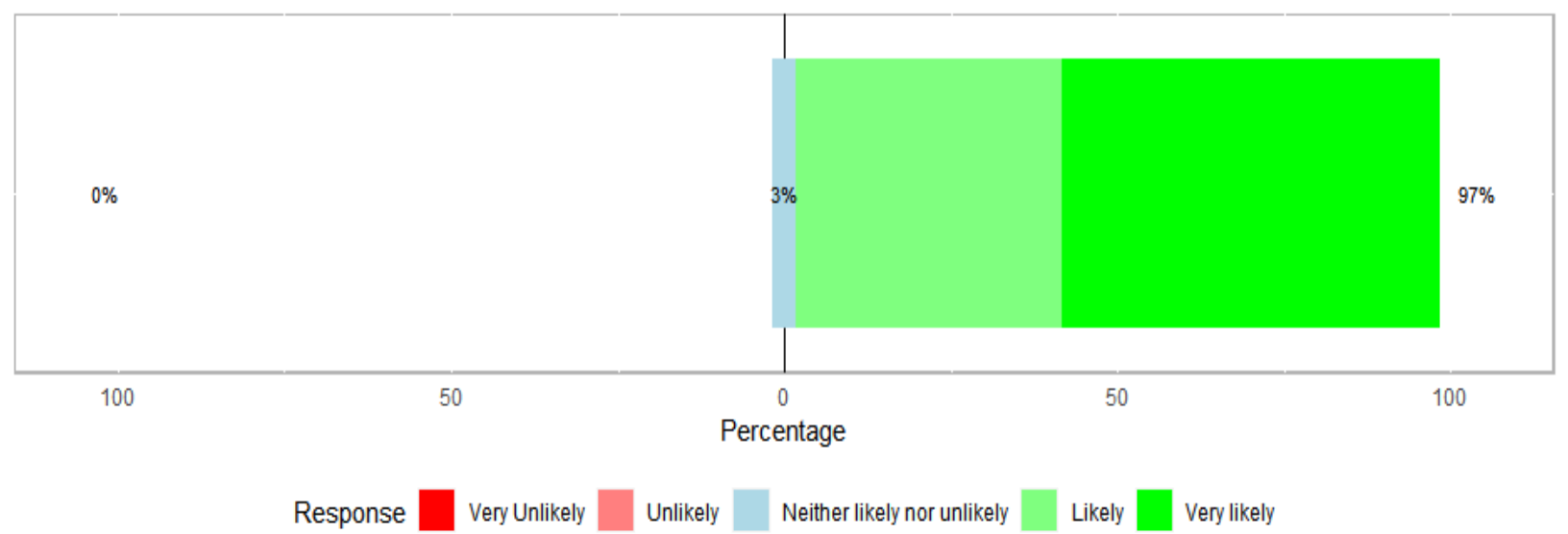




\section{Distribution of Participants per age category (Count \& Percentage)}

30 Responses most participants are aged 25-34 (63.33\%) whereas only (6.67\%) of participants are aged45-54. More details are shown below:
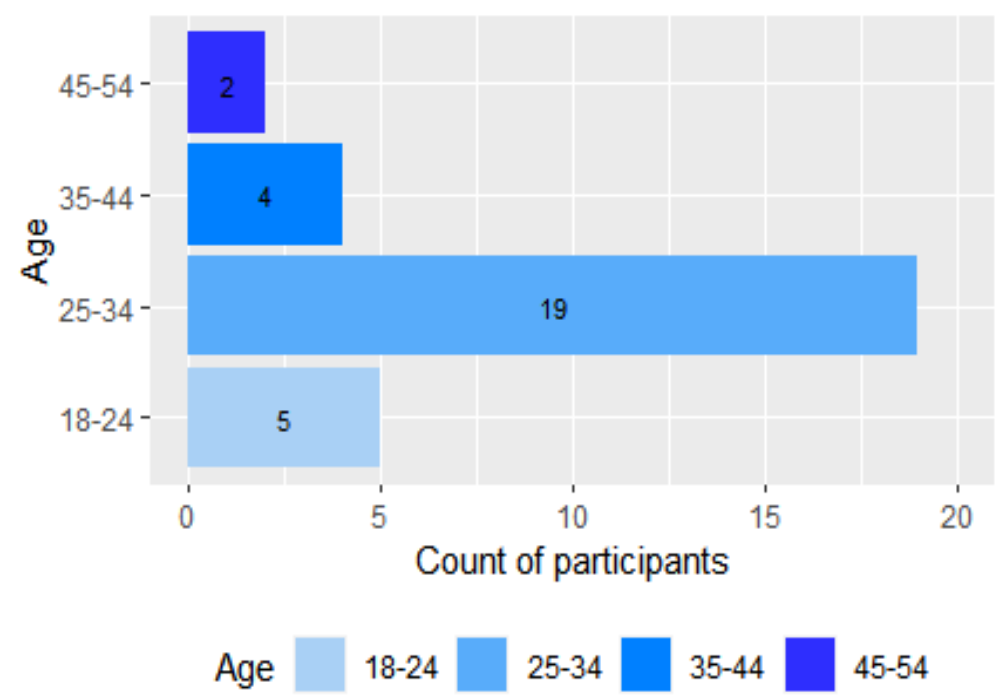

Fig.7

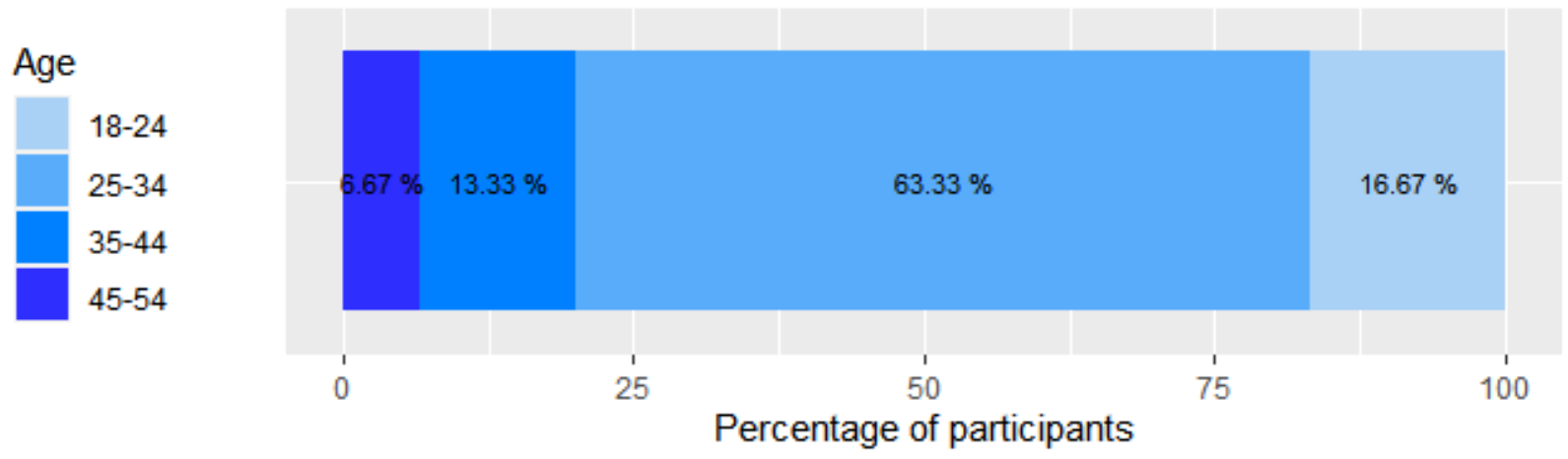

Fig.8

\section{Main Findings}

Overall, Participants have reporteda clearly positive feedback regarding all questions; based on the relevant Likert rating scale. However, a minority of respondents have reported a negative/uncertain feedback regarding some of the questions. Below are more details:

$3 \%$ of participantswere not satised regarding each of the following questions

- Do you think this visualization is intelligible

- Would you buy or interact with this system in future

- Would you recommend this system to a friend

$13 \%$ of participants were uncertain regarding each of the following questions

- Do you think this visualization is intelligible

- How likely do you think that this system is useful $10 \%$ of participants were uncertain regarding the following question- item would you buy or interact with this system in future of participants were uncertain regarding the following

\section{Conclusion and Future Research Directions}

On the off chance that such a gadget as was proposed towards the start of this venture was developed, its applicability would be enormous. Evaluating the $\mathrm{CO} 2$ is of principal significance and research right now proceeds regardless of the difficulties encountered. The current form of the framework can only quantify heartbeat on to the mobile application. On the other hand there is space for the improvements. 
Barely any different parameters like glucose levels, BMI (Body Mass Index), waist circumference, internal heat level and so forth., can also be estimated. Then the complete health status of patient will be recorded and readily available on the mobile application. With the end goal that it will be a lot simpler to the patient to screen the advancement of health now.

This paper emphasizes on improving the monitor of human heath using Arduino and mobile application. The more important it is to get the accurate patient's health data on time, it is also more important to protect the data as security is the important concern and a challenging factor for protecting the data from intruders. Max32102 sensor is used to collect data of heartbeat. As the saying goes "Prevention is better than cure", the early detection of the problem will help the patients as well as the doctors to take first aid well in advance.

\section{References}

[1] Couderc, J. P. (2010, September). The telemetric and Holter ECG warehouse initiative (THEW): a data repository for the design, implementation and validation of ECG-related technologies. In 2010 Annual International Conference of the IEEE Engineering in Medicine and Biology (pp. 6252-6255). IEEE

[2] IOT systems and its impacts. https://internetofthingsagenda.techtarget.com/definition/Internet-of-Things-IoT

[3] Bell, C. (2005). Understanding contemporary pulse oximetry. GE Healthcare International web journal, Clinical Paper, GE Healthcare and New Cork University School of Medicine.

[4] Siegfrid, K., Friedemann, N., Siegfrid, F., Anton, B., Eberhard, M., \& Dietmar, M. (1997). A new family of sensors for pulse oximetry. Hewlett-Packard J, 48, 1-17.

[5] The chip on MAX30102 has body heat sensor https://www.elprocus.com/temperature-sensors-types-workingoperation/

[6] Guyton, A. C., \& Hall, J. E. (2006). Textbook of Medical physiology 11 th Edition. Rio de.

[7] Arduino- promini ATmega32 https://www.theengineeringprojects.com/2018/06/introduction-to-arduino-promini.html.

[8] Hanlon, P. (2014). Pulse Oximetry in the patient's hands: as telemedicine opportunities expand, companies and respiratory care professionals are developing mobile health devices that connect more patients with the benefits of pulse oximetry. RT for Decision Makers in Respiratory Care, 27(2), 20-23.

[9] Likert scale: definition, examples \& analysis. https://study.com/academy/lesson/likert-scale-definition-examplesanalysis.html.

[10] Customer satisfaction and attitude scales. https://business.critizr.com/en/blog/customer-satisfaction-and-attitudescales 